\title{
Editorial
}

\section{Surveillance, Reporting, Automation, and Interventional Epidemiology}

\author{
John P. Burke, MD
}

A commonly accepted definition of public health surveillance is "the ongoing, systematic collection, analysis, and interpretation of health data essential to the planning, implementation, and evaluation of public health practice, closely integrated with the timely dissemination of these data to those who need to know."1 The scientific basis for surveillance as a part of a hospital infection control program was established by the landmark Study on the Efficacy of Nosocomial Infection Control (SENIC). The SENIC project was born out of the frustration to justify infection control programs that had been instituted in thousands of hospitals in the 1960s and 1970s. ${ }^{2}$ The SENIC investigators recognized (and wished to measure) how the surveillance activity itself could influence patient care through the Hawthorne effect (ie, how healthcare workers may alter their practice when they see that someone is watching and is interested in how they are caring for patients). ${ }^{3}$

At the time of the first SENIC survey in 1976, most (86\%) of the 1,518 infection control practitioners spent half or more of their time in surveillance activities. ${ }^{4}$ The SENIC project found that reduced rates of nosocomial infections were strongly associated with intensive surveillance activities, although at least moderate levels of control activities were also required for effectiveness. ${ }^{5}$ The SENIC study design was thorough, ambitious, and technically sophisticated, but it was based on retrospective chart reviews in a large sample of hospitals and not, as sometimes stated, on a controlled trial. ${ }^{6}$

The incremental and slow development of definitions and methods for surveillance of healthcare-associated infections was reviewed by Hierholzer at the Third Decennial International Conference on Nosocomial
Infections and cited as "sobering to those who are impatient for quick answers in health care evaluation." Hierholzer further pointed out that "the defined elements in [the SENIC] data bank are no longer relevant to the state of the art in the field." ${ }^{m}$ In 2000, the Centers for Disease Control and Prevention (CDC) National Nosocomial Infections Surveillance (NNIS) System, the only national source of data regarding the incidence of, nature of, and trends in hospital infections, reported findings that also supported the benefits of surveillance: during the period 1990-1999, risk-adjusted infection rates decreased for all three body sites (ie, respiratory tract, urinary tract, and bloodstream) monitored in intensive care units. ${ }^{8}$ The NNIS System investigators acknowledged, however, the limitations of these findings, but believed that patient care personnel did perceive value in the data and altered their behavior in ways that may have reduced the rate of nosocomial infections in NNIS System hospitals. ${ }^{1}$

A clear understanding of the purposes of surveillance is a good starting place. Enthusiasts for surveillance argue that without ongoing surveillance, we will not know the effectiveness of our infection control efforts. This may be overreaching in view of the inability of surveillance to account for the multiple reasons behind secular changes in infection rates. On the other hand, others believe that the detection and management of outbreaks is the primary target of surveillance. ${ }^{9}$ In the United States today, the minimal estimate of the epidemic nosocomial infection burden is at least 40,000 cases annually, and the maximal estimate is as much as five times that figure..$^{10}$ Outbreaks clearly represent threats to patient safety and fall within the concerns expressed in the recent Institute of Medicine (IOM) report "To Err Is Human."11 
The NNIS System's working hypothesis is that ongoing surveillance measures the endemic-disease rate, which accounts for $90 \%$ to $95 \%$ of all hospital infections. ${ }^{1}$ The NNIS System uses computer entry from reporting hospitals, but has been plagued by underreporting, delayed reports, and labor-intensive methods that have led to its dropping the hospital-wide surveillance component in favor of reporting infections in selected high-risk procedures or units. The impact of these changes, both positive and negative, on the detection of outbreaks has probably not been assessed but is likely important. For instance, the Intensive Care Antimicrobial Resistance Epidemiology (ICARE) project has enhanced the NNIS System's ability to detect epidemic antibiotic resistance. ${ }^{12}$ Following the CDC's lead, many hospitals have adopted targeted surveillance methods or various schemes for rotating surveillance. In consequence, outbreak detection in most hospitals is, in large part, a passive system that depends on voluntary reporting by alert employees. ${ }^{13}$

The current status of surveillance activities in hospitals is largely unknown, although surveillance is now considered the "cornerstone" of infection control programs. ${ }^{13}$ The shortcomings of existing patient record systems have led to the development of unique infection control databases in many hospitals that, even if stored and analyzed in departmental computers, require the manual collection, entry, and interpretation of individual patient reports. Denominator data can often be downloaded from other hospital computer systems, but the interpretation of numerators still requires human effort to link clinical and laboratory findings. Nor has the uniform hospital discharge data set provided a solution to this problem because of underreporting and frequent lack of critical information in the patient records. The authors of a recent review of cost-benefit and effectiveness of surveillance methods estimated that an infection control program should devote at least $25 \%$ of its effort to surveillance. ${ }^{13}$

The computer-based patient record has become one of the hoped for innovations that could lead to higher quality of data sets for infection control. In 1991, the IOM proclaimed that the computer-based patient record is an essential technology for health care and recommended steps that it believed would lead to the goal of widespread computerbased patient record utilization within a decade, a milestone that has now passed. ${ }^{14}$ The 1999 IOM report further recommended computerized physician order entry and pharmaceutical software as strategies to improve medication safety. However, progress toward the goal of computerizing patient records remains elusive and computerbased patient records have been implemented in only $12 \%$ to $13 \%$ of U.S. hospitals. ${ }^{15}$ Although the speed and storage capacity of computers have increased dramatically and the Internet has enabled instant dissemination of medical knowledge, existing computer-based patient record systems remain limited in their scope. For example, the Veterans Administration system, where 140 hospitals now run a paperless patient record system, does not provide decision support capabilities or tools for epidemiology. ${ }^{16}$
The reason for this disappointing gap between promise and reality is not physicians' reluctance to use computers, but rather technical issues related to coding of medical data. ${ }^{15}$

The modern trends of targeted surveillance and the collection of reports from computerized administrative and clinical databases should cause reflection that the "illdefined but quite real charisma factor" ${ }^{2}$ of infection control professionals assessed in SENIC may now be diminished if not entirely lost. Will automation eliminate the Hawthorne effect of shoe-leather epidemiology? The SENIC project, after all, demonstrated the value of "pencil and paper" surveillance derived from ward visits by infection control professionals. The promise of automation is accompanied by the expectation that the time saved in hospital surveillance will yield increased time for interventions that will actually prevent infections. ${ }^{17}$ But how realistic is this expectation? Will automation simply lead to the further downsizing of infection control programs as surveillance becomes more efficient? ${ }^{18}$ The answers are not clear.

The rapidly increasing need for site-specific infection rates adjusted for hospital services, high-frequency, highrisk procedures, and device-days (ventilators, bladder catheters, and intravascular catheters, including central venous catheters, arterial catheters, dialysis catheters, and the like) is eminently justified by the growing knowledge base of infection control. However, even this level of risk adjustment is criticized as too superficial by other health services researchers. Have we expanded the scope of surveillance too far, or is automation the answer? Our concept of surveillance owes much to the thinking of Alexander Langmuir, who cautioned against the tendency to expand its scope too far and advised that the "actual performance of the [epidemiologic] study should be recognized as a function separate from surveillance." ${ }^{19}$ Langmuir acknowledged that, for public health surveillance at least, the methods are intrinsically crude, inaccurate, and incomplete, and he encouraged us to "use the term wisely and recognize its proper limitations." ${ }^{19}$ Platt has pointed out that surveillance of just one type of infection-surgical-site infection-would require the full-time efforts of all existing infection control professionals and that more efficient computer-driven approaches appear to be the only practical solution..$^{20}$ Adding to these dilemmas, advocates for patient safety are calling for increased voluntary reporting and root-cause analysis of preventable adverse events, including infections. ${ }^{6}$ As Eickhoff wrote (in another context), "Verily, we have reaped a whirlwind."21

Innovative approaches to surveillance are needed and can be developed only at the local level in individual healthcare systems or in collaboratives, often under the pressure of limited resources. The article by Bouam et al. in this issue of Infection Control and Hospital Epidemiology is an example of cooperation between an infection control department and the hospital's information department. ${ }^{22}$ Platt has reminded us that we are unlikely ever to have vastly greater resources and the prospect of better information systems is uncertain. ${ }^{20}$ Nor should surveillance be limited to simply automating traditional surveillance meth- 
ods; computerized event monitoring is increasingly being used to develop population-based decision support tools that can "trigger" epidemiologic investigations, ${ }^{23}$ and "virtual surveillance" using the techniques of data mining of clinical microbiology results can uncover many small outbreaks that would otherwise go undiscovered and that would be, at least theoretically, amenable to intervention. ${ }^{24}$ Traditional surveillance strategies require that outcomes of interest be known before monitoring begins, whereas data mining can detect new and unexpected patterns. ${ }^{25}$ Long outmoded techniques for surveillance may also merit reexamination; for example, Ford-Jones et al. have used sentinel sheets to encourage voluntary reporting of infections in newborns. ${ }^{26}$ We can expect that such efforts and automation will create the need for more, not fewer, infection control professionals. Time saved by automating surveillance will lead to the need for more human work, not less, to manage still more data and answer yet more questions.

\section{REFERENCES}

1. Gaynes RP, Richards C, Edwards J, et al. Feeding back surveillance data to prevent hospital-acquired infections. Emerg Infect Dis 2001;7:295-298.

2. Eickhoff TC. General comments on the Study on the Efficacy of Nosocomial Infection Control (SENIC Project). Am J Epidemiol 1980;111:465-469.

3. Haley RW, Quade D, Freeman HE, et al. Study on the Efficacy of Nosocomial Infection Control (SENIC Project): summary of study design. Am J Epidemiol 1980;111:472-485.

4. Haley RW, Shachtman RH. The emergence of infection surveillance and control programs in US hospitals: an assessment, 1976. Am J Epidemiol 1980;111:574-591.

5. Haley RW, Culver DH, White JW, et al. The efficacy of infection surveillance and control programs in preventing nosocomial infections in US hospitals. Am J Epidemiol 1985;121:182-205.

6. Leape LL. Reporting of adverse events. N Engl J Med 2002;347:16331638.

7. Hierholzer WJ. Health care data, the epidemiologist's sand: comments on the quantity and quality of data. Am J Med 1991;91 (suppl 3B):3B-21S3B-26S.

8. Centers for Disease Control and Prevention. Monitoring hospitalacquired infections to promote patient safety: United States, 1990-1999. MMWR 2000;49:149-153.
9. Gaunt PN, Phillips I. Computers and hospital infection. J Hosp Infect 1987;9:106-109.

10. Brown SM, Benneyan JC, Theobald DA, et al. Use of binary cumulative sums and moving averages in nosocomial infection cluster detection. Emerg Infect Dis 2002. Available at www.cdc.gov/ncidod/EID/ vol8no12/01-0514.htm.

11. Institute of Medicine. To Err Is Human. Washington, DC: National Academy Press: 1999.

12. Fridkin SK, Steward CD, Edwards JR, et al. Surveillance of antimicrobial use and antimicrobial resistance in United States hospitals: Project ICARE phase 2. Clin Infect Dis 1999;29:245-252.

13. Obasanjo $O$, Perl $T$. Cost-benefit and effectiveness of nosocomial surveillance methods. Curr Clin Top Infect Dis 2001:21:391-406.

14. Institute of Medicine. The Computer-Based Patient Record: An Essential Technology for Health Care. Washington, DC: National Academy Press; 1991.

15. Benson $T$. Why general practitioners use computers and hospital doctors do not: Part 1. Incentives; Part 2. Scalability. BMI 2002;325:10861093.

16. Best WR, Khuri SF, Phelan M, et al. Identifying patient preoperative risk factors and postoperative adverse events in administrative databases: results from the Department of Veterans Affairs National Surgical Quality Improvement Program. J Am Coll Surg 2002;194:257-266.

17. Burke JP, Pestotnik SL. Antibiotic resistance: the combat zone. Curr Opin Infect Dis 1998;11:441-443.

18. Calfee DP, Farr BM. Infection control and cost control in the era of managed care. Infect Control Hosp Epidemiol 2002;23:407-410.

19. Langmuir $\mathrm{AD}$. Evolution of the concept of surveillance in the United States. Proc R Soc Med 1971;64:681-684.

20. Platt R. Progress in surgical-site infection surveillance. Infect Control Hosp Epidemiol 2002;23:361-363.

21. Eickhoff TC. Nosocomial infections-a 1980 view: progress, priorities and prognosis. $\mathrm{Am} \mathrm{J} \mathrm{Med} \mathrm{1981;70:381-388.}$

22. Bouam S, Girou E, Brun-Buisson C, Karadimas H, Lepage E. An intranet-based automated system for the surveillance of nosocomial infections: prospective validation compared with physicians' selfreports. Infect Control Hosp Epidemiol 2003;24:51-55.

23. Jenders RA, Shah A. Challenges in using the Arden syntax for computer-based nosocomial infection surveillance. $J$ Am Med Inform Assoc 2002;9(suppl 6):S98-S101.

24. Peterson LR, Brossette SE. Hunting health care-associated infections from the clinical microbiology laboratory: passive, active, and virtual surveillance. J Clin Microbiol 2002;40:1-4.

25. Brossette SE, Sprague AP, Hardin JM, Waites KB, Jones WT, Moser SA Association rules and data mining in hospital infection control and public health surveillance. J Am Med Inform Assoc 1998;5:373-381.

26. Ford-Jones EL, Mindorff CM, Pollock E, et al. Evaluation of a new method of detection of nosocomial infection in the pediatric intensive care unit: the infection control sentinel sheet system. Infect Control Hosp Epidemiol 1989;10:515-520. 